\title{
Thermally Latent Water in a Polymer Matrix
}

\author{
Makoto Gemmei-Ide*, Atsushi Ohya, and Hiromi Kitano*
}

\begin{abstract}
Department of Environmental Applied Chemistry, Graduate School of Science and Engineering, University of Toyama, 3190 Gofuku, Toyama 930-8555, Japan
\end{abstract}

(H.K.) E-mail: kitano@eng.u-toyama.ac.jp; Tel: +81-76-445-6868; Fax: +81-76-445-6703.

(M. G.-I.) E-mail: ide@eng.u-toyama.ac.jp; Tel: +81-76-445-6870; Fax: +81-76-445-6703.

\begin{abstract}
Possible phase transition of water in polymer-water systems has been understood (prejudiced) to be only either freezing/melting or nothing, without relation to whether the polymer was soluble or not in water or whether its water content was high or low. This general understanding of water structure has been structured on the basis of calorimetric analyses such as differential scanning calorimetry, DSC. DSC is one of the most frequently used methods to analyze the water structure in polymer-water systems because the data obtained are relatively easy to interpret. This easiness of interpretation, however, can be accepted only if based on the understanding stated above. Unexpectedly, results of the infrared spectroscopic analysis presented here completely denied the general understanding and provided definite evidence of the existence of all phase transitions among the three states of water in a polymer solid. Furthermore, the impossibility of detection of condensation, deposition, sublimation and vaporization by calorimetric analysis was revealed.
\end{abstract}

Keywords: DSC / IR spectroscopy / Non-freezing water / Phase transition

\section{Introduction}

Differential scanning calorimetry, DSC, is one of the most frequently used methods to analyze the physicochemical properties of water, that is, water structure, in polymer-water systems. It is not an exaggeration to say that the picture of water structure is dominated by calorimetric analysis. This is because the data obtained are relatively easy to interpret. Water in this method is categorized into three types, free water which melts at $\sim 273 \mathrm{~K}$, freezing bound (FB) water which melts below $273 \mathrm{~K}$, and non-freezing bound (NFB) water which does not freeze even below $173 \mathrm{~K} .{ }^{1}$ The former two are detected by DSC as exo/endothermic signals and their amounts are estimated on the basis of an equality assumption between the freezing/melting enthalpies, $\Delta_{\mathrm{f}} \mathrm{H}$, of pure water at $273 \mathrm{~K}$ and those of the two waters. NFB water, in contrast, is defined as the difference between the actual amount of water and the amount of water detected by DSC. This means that NFB water is the one undetected by DSC.

A general understanding of the water structure based on DSC was that the more water content decreases, the more the ratio of NFB water increases, and finally all of the water becomes NFB water. Although this understanding was based mainly on study of water-soluble polymer systems, it was regarded to be adaptable to non-water-soluble polymer systems. All of the water sorbed into non-water-soluble polymers by vapor sorption was also considered to be NFB water. Water in a poly $(n-$ butylacrylate), PBA, with water content of $0.44 \mathrm{wt} \%$ hydrated by vapor sorption has been expected to be NFB water. In fact, the expectation has been demonstrated by DSC. ${ }^{2}$

Figure 1 showing a DSC curve of the hydrated PBA, however, definitely conflicts with the literature: an exothermic peak at $\sim 230 \mathrm{~K}$ on cooling, and an endothermic peak at $\sim 270 \mathrm{~K}$ and an endothermic broad component above $\sim 245 \mathrm{~K}$ on heating were clearly observed. The value of $\Delta_{\mathrm{f}} H$, (hatched area) was given in the figure and the ratios of NFB water were calculated to be $57 \%$ and $53 \%$ from the cooling and heating DSC curves, respectively.

The discrepancy between the literature and the present results might not be a serious problem because the reason for the discrepancy is only the difference in sensitivity of apparatus. The aim of this report is not to discuss the changing picture of water structure by the apparatus but to show the existence of water that cannot be detected in principle by calorimetric 
analysis, that is, thermally latent water. Infrared spectroscopy showed evidence of the existence of all types of phase transition among the three states of water. This means that for calorimetric analysis, at least in the case of low water content systems, the heat flow observed does not always indicate the existence of liquid water at physiological $T$ and that the true amount of NFB water cannot be determined.

\section{Experimental Section}

Poly(n-butylacrylate), PBA, was prepared by a conventional radical polymerization of $n$-butylacrylate using $2,2^{\prime}$ azobisisobutylonitrile as a thermal initiator at $348 \mathrm{~K}$ in toluene. The weight-averaged molecular weight and the polydispersity index were determined to be $7.9 \times 10^{4}$ and 1.5 , respectively, by gel permeation chromatography.

Calorimetric analysis of the hydrated PBA, which was shown in the Introduction, was performed by a differential scanning calorimetry (DSC204 F1, Netzsch). The hydrated sample of $11.49 \mathrm{mg}$ placed in an aluminum pan was hermetically sealed. The sample was held at $303 \mathrm{~K}$ for $30 \mathrm{~min}$, cooled to $168 \mathrm{~K}$, held at $168 \mathrm{~K}$ for $30 \mathrm{~min}$, and heated to $303 \mathrm{~K}$. Cooling and heating rates were $5 \mathrm{~K} \cdot \mathrm{min}^{-1}$.

Infrared spectra of the hydrated and dried PBA were recorded on a Spectrum One NTS (Perkin-Elmer) with a resolution of $4 \mathrm{~cm}^{-1}$ and a scanning time of $20 \mathrm{~s}$. Cooling and heating rates were 5 and $4 \mathrm{~K} \cdot \mathrm{min}^{-1}$, respectively. Sample temperature was controlled by an Optistate-DN cryostat (Oxford) connected to a model ITC601 $T$ controller. The spectra of the sorbed water were obtained by subtraction between the spectra of the hydrated and dried samples.

Water content of hydrated samples was measured by the Karl Fisher method. The sample was hydrated by exposing to humid air with a constant relative humidity for 2 weeks. For IR and DSC measurements, the sample was exposed to humid air with $88 \% \mathrm{RH}$ at $310 \mathrm{~K}$ for 2 weeks and its water content was determined to be $0.44 \mathrm{wt} \%$. To determine the enthalpy of water sorption, water contents at various T's and RH's were measured. Based on Henry's law of sorption and van't Hoff plots of Henry's law constant (solubility coefficient), the sorption enthalpy was estimated to be $-42 \mathrm{~kJ} \cdot \mathrm{mol}^{-1}$. These data were given in Supporting Information.

\section{Results and Discussion}

Figure 2 shows the IR spectra for pure water at $298 \mathrm{~K}$, ice $I_{\mathrm{h}}$ at $170 \mathrm{~K}$, and water in hydrated PBA at 298 and $170 \mathrm{~K}$. The spectral difference of the sorbed water at $298 \mathrm{~K}$ to pure water clearly shows the isolated water localizing on hydrogen bonding sites of PBA. Its spectral profile seemed to be the same as that in the literature. ${ }^{2}$ The two peaks at 3640 and 3556 $\mathrm{cm}^{-1}$ might be assigned to water binding to ester oxygen in PBA and the uneven shape at $\sim 3400 \mathrm{~cm}^{-1}$ is thought to be caused by the red shift of $\mathrm{C}=\mathrm{O}$ stretching overtone by hydration. ${ }^{2-4}$ The difference at $298 \mathrm{~K}$ hardly allows us to expect phase transition of the sorbed water, which agrees with the general understanding. However, the spectral similarity of the sorbed water at $170 \mathrm{~K}$ with ice $I_{\mathrm{h}}$ denied the agreement. The DSC results presented here, on the other hand, do not conflict with the IR results. If there was crystal formation by deposition or/and freezing after condensation, the IR result was not strange.

Figure 3 shows $T$ dependence of the IR spectra. During cooling (left panels), the spectra for the sorbed water (panel A) showed a new broad component in the region of $3600-3000 \mathrm{~cm}^{-1}$, and then changed suddenly to an ice-like spectrum below $\sim 234 \mathrm{~K}$. The spectral shape in the lower wavenumber region above $\sim 234 \mathrm{~K}$ was similar to that of liquid water although the two sharp peaks in the higher wavenumber region remained. This indicates condensation of a part of monomolecular water in the polymer matrix and coexistence of liquid water and monomolecular water. $T$ dependence of absorbance of the characteristic peak for ice $I_{\mathrm{h}}$ at $\sim 3280 \mathrm{~cm}^{-1}, A_{3280}$, was plotted against $T$ together with a peak absorbance assigned to the isolated water, $A_{3640}$ (panel C). The $A_{3280}$ value corresponds to the absorbance at a peak around $3280 \mathrm{~cm}^{-1}$ in the $T$ regions where the peak appeared (in the case of PBA, below $234 \mathrm{~K}$ on cooling and below $273 \mathrm{~K}$ on heating; in the case of pure water, below $260 \mathrm{~K}$ on cooling and below $273 \mathrm{~K}$ on heating) and to the absorbance at $3280 \mathrm{~cm}^{-1}$ in other $T$ regions. The value of $A_{3280}$ increased gradually and jumped up at $\sim 234 \mathrm{~K}$, which were synchronized with the gradual decrease and the jumping down of $A_{3640}$, respectively. These spectral changes and the changes in $A_{3280}$ and $A_{3640}$ indicate condensation and freezing at $\sim 234 \mathrm{~K}$.

Two noticeable points are (1) a large amount of NFB water determined by DSC and (2) no DSC signal in the $T$ region of 298-240 K. Assuming no $T$ dependence of absorptivity of $A_{3640}$, the ratio of NFB water was calculated to be at most only $\sim 16 \%$ (The values of $A_{3640}$ at $298 \mathrm{~K}$ and $170 \mathrm{~K}$ were 0.253 and 0.040 , respectively.). In general, the peak absorptivity, not area, increases with decrease in $T$, so that the actual ratio of NFB water should be smaller than $16 \%$. The discrepancy of DSC with IR results might only be caused by the difference in sensitivity to water but cannot be ignored. Furthermore, the "no DSC signal" is strange in view of the IR results because condensation enthalpy of water is very large, $-45 \mathrm{~kJ} \cdot \mathrm{mol}^{-1}$ at $273 \mathrm{~K}$. Here, if condensation in the polymer matrix is regarded to be segregation of water molecules from the polymer matrix, this might be solved by enthalpy of water desorption from the polymer matrix. The desorption enthalpy was estimated to be +42 $\mathrm{kJ} \cdot \mathrm{mol}^{-1}$, which was comparable to the condensation enthalpy in the opposite sign, so that the two heat flows should be canceled out. This explains both DSC and IR results and, at the same time, indicates that detection of the condensation of sorbed water is difficult for calorimetric analysis and that this condensed water is latent water for calorimetric analysis. A similar phenomenon was also observed in the heating process.

During heating (right panels), the spectral intensity of ice in PBA decreased up to $273 \mathrm{~K}$ with maintenance of spectral shape of ice. The decreasing ratio (panel A) was much larger than that for ice $I_{\mathrm{h}}$ (panel B). An acceleration of decrease in 
$A_{3280}$ occurred at $229 \mathrm{~K}$ together with an increase in $A_{3640}$ (panel C). These results are definite evidence of sublimation above $229 \mathrm{~K}$. On the other hand, a DSC signal was observed only above $\sim 245 \mathrm{~K}$. Based on the general interpretation, all of the DSC signals might be assigned to melting of ice. As indicated by IR results, however, this is an erroneous interpretation and most of the ice in PBA was reduced by sublimation. Sublimation enthalpy is very large, $+51 \mathrm{~kJ} \cdot \mathrm{mol}^{-1}$ at $273 \mathrm{~K}$, so that a large difference in enthalpy between cooling and heating processes could be observed. Unexpectedly, a good balance was obtained (Figure 1), which should be explained by the sorption enthalpy of the sublimated water molecules to the polymer matrix. This is the opposite case of condensation. The sublimated water is also latent water for calorimetric analysis.

Evidence of vapor deposition after freezing and vaporization after melting was also obtained. After freezing and melting, an immediate linear relation of the value of $A_{3280}$ to $T$ was observed in the case of pure water but not in the case of the sorbed water.

\section{Conclusion}

IR spectroscopic results indicated that the general understanding of water sorbed into a non-water-soluble polymer by vapor sorption was not always acceptable: the sorbed water was able to be condensed, crystallized, and deposited during cooling and to be sublimated, fused, and vaporized during heating. These phenomena have been spectroscopically observed in other polymers. ${ }^{5-7}$ Among them, condensation, deposition, sublimation, and vaporization have been hindered by the prejudice that DSC signals below $273 \mathrm{~K}$ should solely be attributed to freezing/melting. This misunderstanding, however, is understandable because the heat flow with the four transitions might not be large enough to expect them to be due to the sorption/desorption enthalpies. These findings not only give a new aspect of water in non-water-soluble polymers but also indicate the need for reconsideration of the general understanding of water in aqueous polymer solutions.

Acknowledgments. This work was supported by Grant-in-Aids for Young Scientists (B20750175) and for Scientific Research on Innovative Areas (20106007) from MEXT. The authors are grateful to Bruker AXS for the measurement of DSC.

Supporting Information Available: water contents of sample at various conditions. This information is available free of charge via the Internet at http://pubs.acs.org.

\section{References}

(1) Jhon, M. S.; Andrade, J. D. J. Biomed. Mater. Res. 1973, 7, 509.

(2) Morita, S.; Tanaka, M.; Ozaki, Y. Langmuir 2007, 23, 3750.

(3) Kusanagi, H.; Yukawa, S. Polymer 1994, 35, 5637.

(4) Sutandar P.; Ahn, D. J.; Franses, E. I. Macromolecules 1994, 27, 7316.

(5) Gemmei-Ide, M.; Kitano, H. J. Phys. Chem. B 2008, 112, 2764.

(6) Gemmei-Ide, M.; Kitano, H. J. Phys. Chem. B 2008, 112, 12863.

(7) Gemmei-Ide, M.; Kitano, H. J. Phys. Chem. B 2008, 112, 13499.

\section{Figure Captions}

Figure 1. DSC curves of hydrated PBA with water content of $0.44 \mathrm{wt} \%$ at cooling and heating rates of $5 \mathrm{~K} \cdot \mathrm{min}^{-1}$. (A) All temperature region; (B) magnified cooling curve; (C) magnified heating curve.

Figure 2. IR spectra for pure water and water sorbed into PBA. Solid line, sorbed water at $298 \mathrm{~K}$; dotted broken line, sorbed water at $170 \mathrm{~K}$; broken line, pure water at $298 \mathrm{~K}$; dotted line, pure water (ice $I_{\mathrm{h}}$ ) at $170 \mathrm{~K}$.

Figure 3. $T$ dependences of IR spectra. (A) Sorbed water in PBA; (B) pure water; (C) $T$ dependence of the values of $A_{3280}$ and $A_{3640}$. Open circle, sorbed water; filled circle, pure water. 

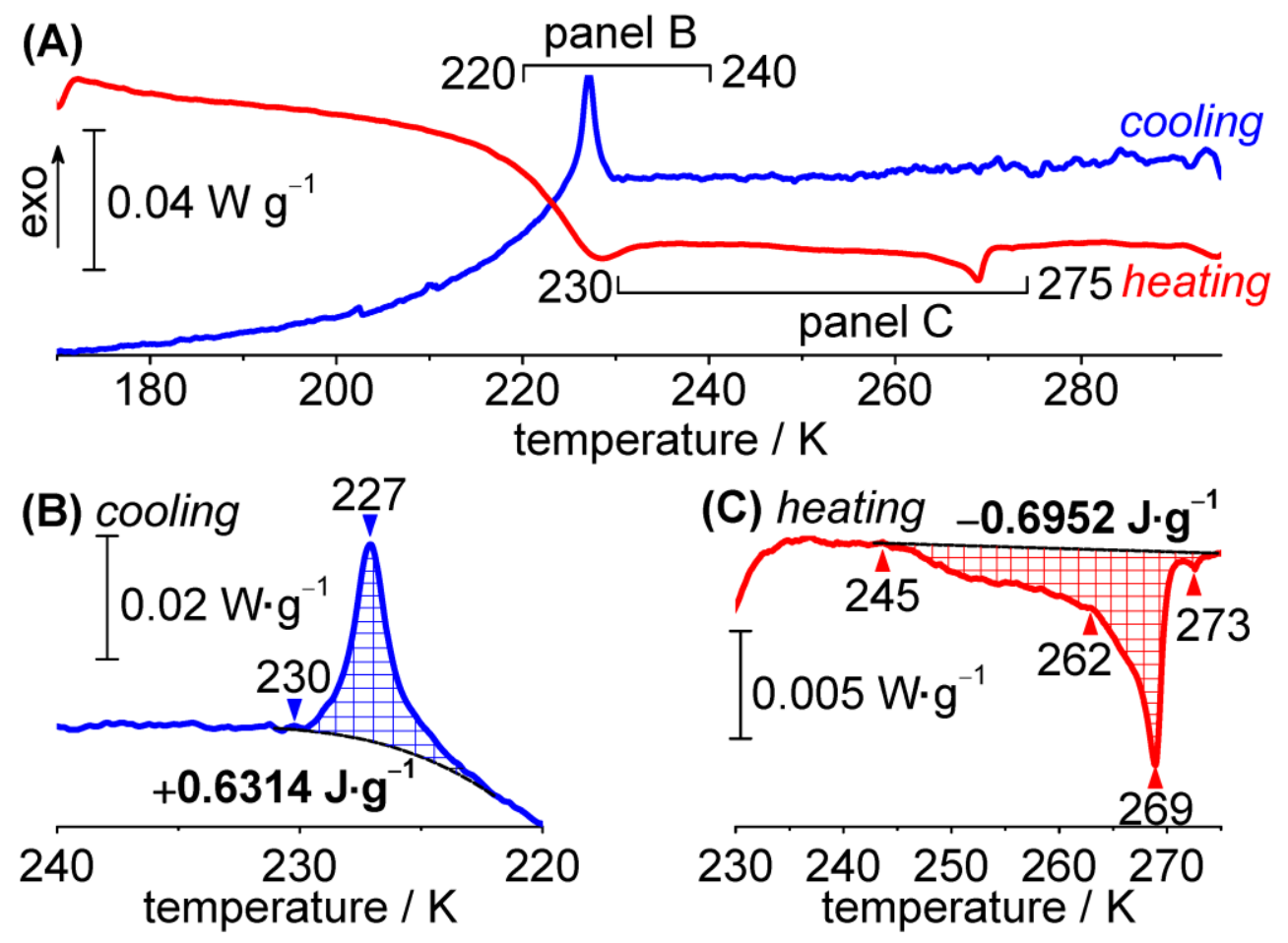

Figure 1. Gemmei-Ide et al. 


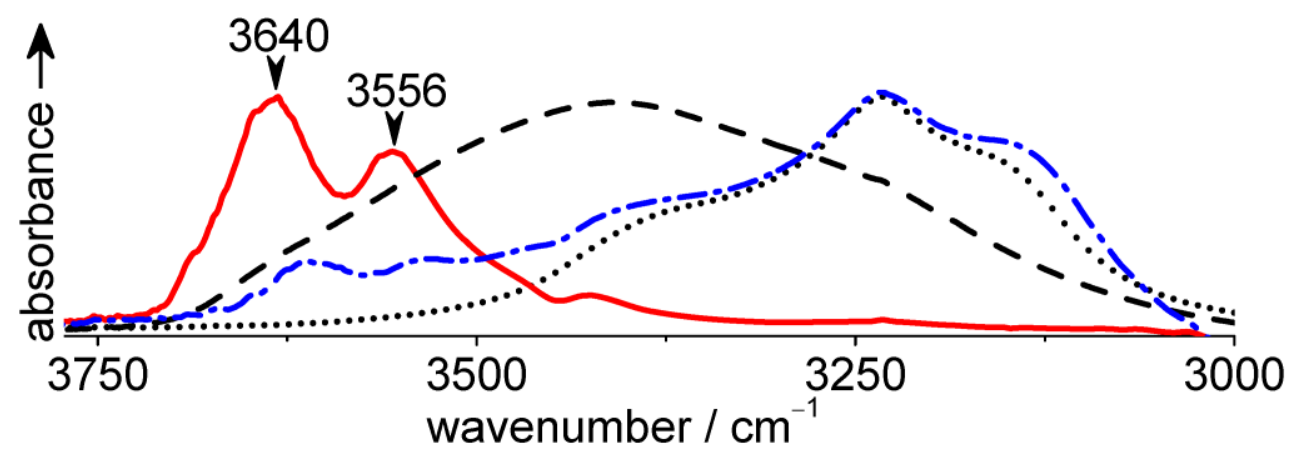

Figure 2. Gemmei-Ide et al. 
cooling process

heating process

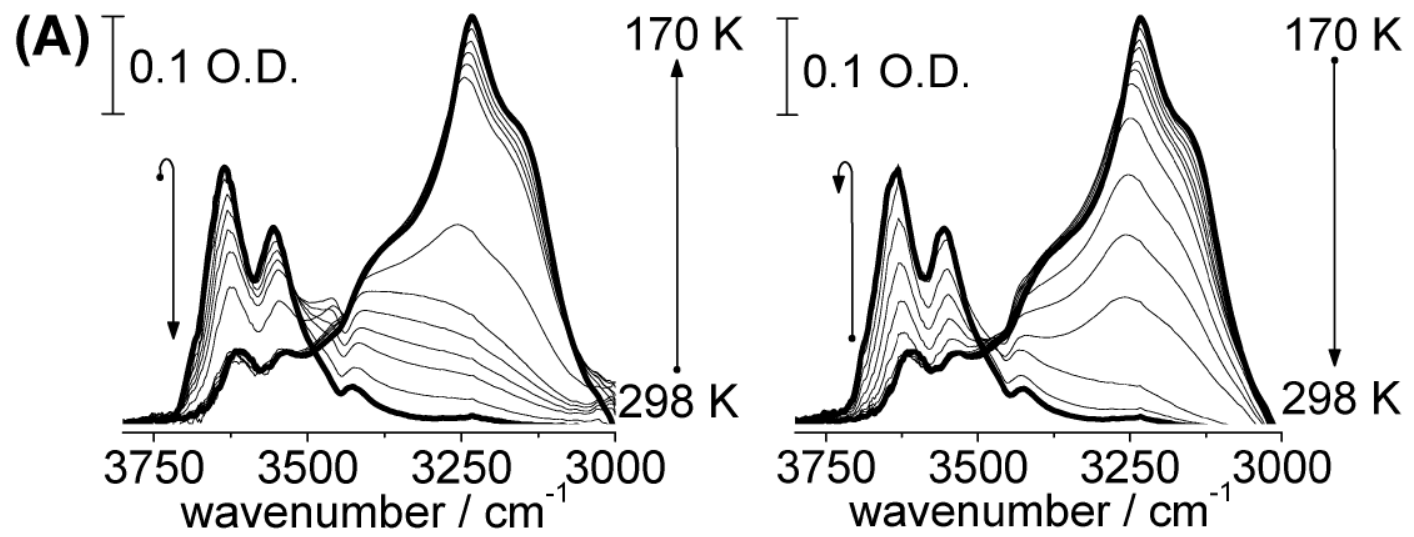

(B)

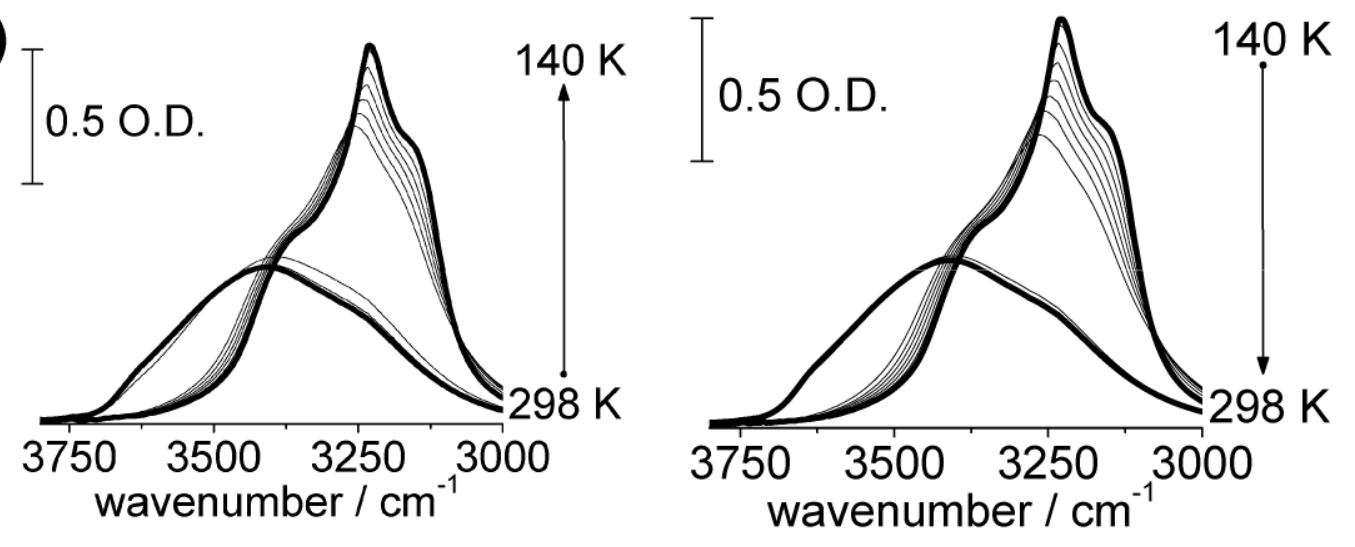

(C)

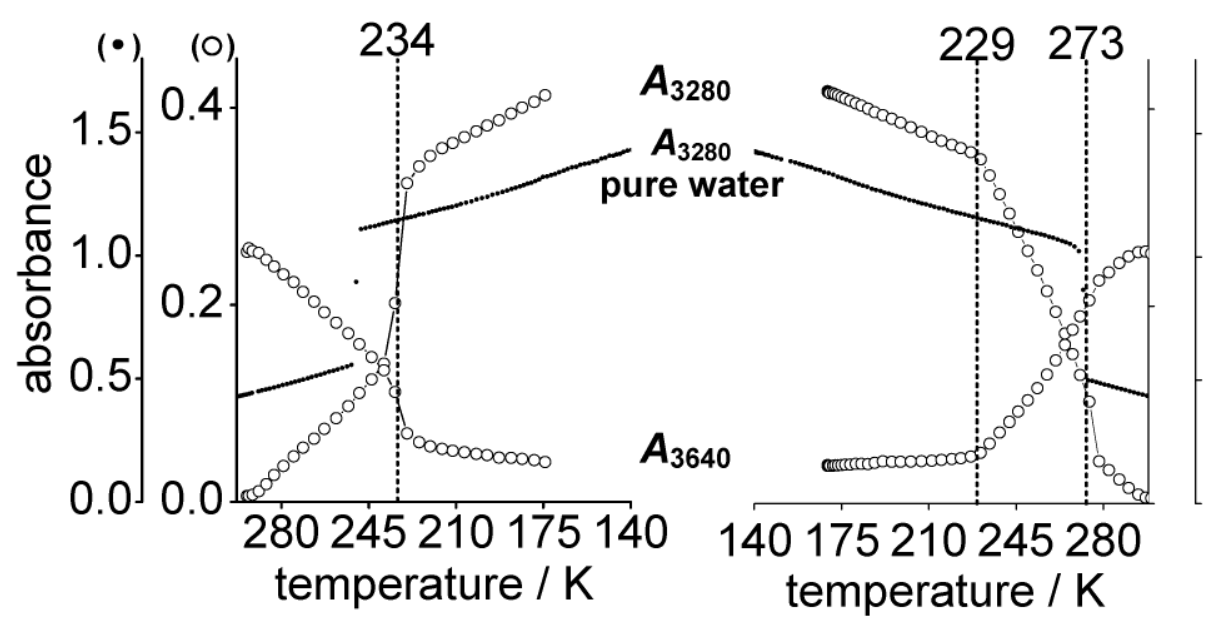

Figure 3. Gemmei-Ide et al. 
TOC Image

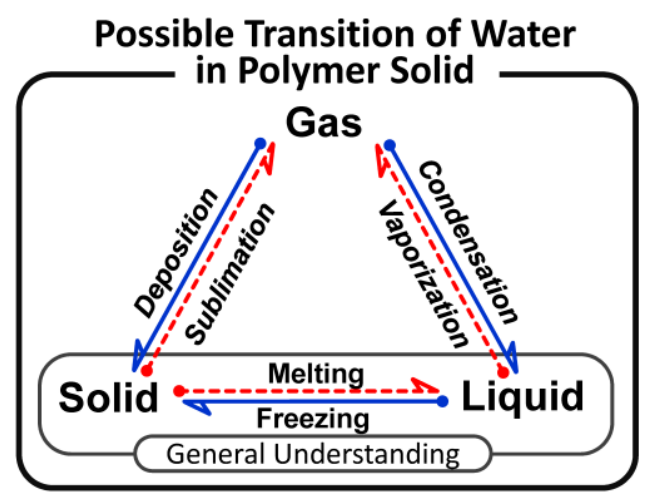

